\title{
Long term exposure to $\gamma$-rays induces radioresistance and enhances the migration ability of bladder cancer cells
}

\author{
GUANGMIN MAO $^{1}$, YE YAO ${ }^{2}$ and ZHAOLU KONG ${ }^{1}$ \\ ${ }^{1}$ Department of Radiobiology, Institute of Radiation Medicine, Fudan University, Shanghai 200032; \\ ${ }^{2}$ Department of Radiation Oncology, Huadong Hospital, Fudan University, Shanghai 200040, P.R. China
}

Received January 17, 2018; Accepted October 10, 2018

DOI: $10.3892 / \mathrm{mmr} .2018 .9605$

\begin{abstract}
In Western countries, bladder cancer (BCa) is one of the most common types of malignancy, with highest incidence rate among urinary system malignancies. At present, as a strategy to preserve the quality and function of the bladder tissues, external-beam radiotherapy has been applied to an increasing number of patients with $\mathrm{BCa}$, particularly the elderly and those in poor health. The onset of acquired radioresistance (ARR) during radiotherapy notably reduces the effectiveness of radiotherapy; however, the cause of ARR is unclear and remains untreatable. In order to investigate the mechanism of ARR, clinical fractionated irradiation was mimicked in vitro to obtain radioresistant $\mathrm{BCa}$ cells, 5637R, in the present study. Compared with the parental cell line, 5637R cells exhibited an increased distribution of S phase cells, enhanced migration ability and elevated levels of phosphorylated-STAT3, as determined using flow cytometry, cell migration analysis and western blotting, respectively. Furthermore, 5637R cells exhibited notable increases in extracellular signal-regulated kinase activation in response to ionizing radiation (IR) compared with 5637 cells. In addition, $5637 \mathrm{R}$ cells showed an early $\mathrm{G}_{2} / \mathrm{M}$ arrest within $8 \mathrm{~h}$ post-IR $(18.23 \pm 3.85$ vs. $6.42 \pm 3.82 \%$, IR cells vs. control cells), whereas 5637 cells were arrested in $\mathrm{G}_{2} / \mathrm{M}$ phase $24 \mathrm{~h}$ post-IR $(19.26 \pm 2.21$ vs. $12.36 \pm 4.45 \%$, IR cells vs. control cells). Overall, the present study suggested that phosphorylated STAT3 may be a potential biomarker to predict radioresistance and tumor recurrence in patients with $\mathrm{BCa}$ following conventional radiotherapeutic intervention. In addition, co-treatment with ERK inhibitor may be a viable approach to increase the anticancer efficacy of radiotherapy in patients with ARR; however, further investigation is required.
\end{abstract}

Correspondence to: Dr Zhaolu Kong, Department of Radiobiology, Institute of Radiation Medicine, Fudan University, 2094 Xietu Road, Shanghai 200032, P.R. China

E-mail: kongzhaolu@fudan.edu.cn

Key words: bladder cancer, migration, fractioned irradiation, radiosensitivity, mitogen-activated protein kinases

\section{Introduction}

Bladder cancer (BCa) is one of the most common malignancies of the genitourinary tract; hematuria is often the initial symptom observed. According to the estimates of the American Cancer Society, BCa has the highest rate of incidence of the urinary system in the United States (1). In 2017, 79,030 novel cases of BCa $(60,490$ male and 18,540 female) and 16,870 mortalities due to $\mathrm{BCa}(12,240$ male and 4,630 female) in US were reported (1). Cytology and certain urine-based markers, including nuclear matrix protein 22 (2) and surviving $(3,4)$, have roles in monitoring individuals at high risk of developing BCa. Additionally, recent research has demonstrated that cigarette smoking (5) and imbalances in sex hormones (6) may be potential risks for the development of $\mathrm{BCa}$.

Radical cystectomy (RC) and radiotherapy, either with or without chemotherapy, are two options of definitive therapy for localized muscle-invasive $\mathrm{BCa}$. Radical radiotherapy alone is not recommended as a first-line therapy for $\mathrm{BCa}$ as the side-effect profile, recurrence and mortality rates are higher than that of $\mathrm{RC}$, with or without chemotherapy (7). Additionally, external-beam radiotherapy is an important method of preserving the quality and function of the bladder tissue in the elderly, and in patients with poor health, or normal upper urinary tract and adequate bladder capacity (8). A British randomized trial reported that there were no statistically significant differences in survival between patients receiving radical surgery and radical radiotherapy (9), suggesting that radiotherapy may be widely applied in the future for the treatment of $\mathrm{BCa}$. Therefore, understanding the biological response of $\mathrm{BCa}$ tissue to radiotherapy may improve the efficacy and side effect profile of radiotherapy. By mimicking the clinical fractionated irradiation (FI) program in $\mathrm{BCa}$ cells, the present study aimed to investigate phenotypic alterations of $\mathrm{BCa}$ cells.

\section{Materials and methods}

Cell culture and treatment. A human BCa cell line, 5637, was purchased from the Cell Bank of Type Culture Collection of the Chinese Academy of Science (Shanghai, China) and maintained in Dulbecco's modified Eagle's medium (DMEM; high glucose; Invitrogen; Thermo Fisher Scientific, Inc., Waltham, MA, USA) containing $10 \%$ fetal bovine serum (FBS; Invitrogen; Thermo Fisher Scientific, Inc.), 100 U/ml 
penicillin and $100 \mathrm{U} / \mathrm{ml}$ streptomycin (Sigma-Aldrich; Merck KGaA, Darmstadt, Germany) under a humidified atmosphere with $5 \%$ carbon dioxide at $37^{\circ} \mathrm{C}$. Cells were irradiated at room temperature in ambient air using a ${ }^{137} \mathrm{Cs}$ source $(\gamma$-ray; Nordion, Inc., Ottawa, Canada) at a dose rate of $0.79 \mathrm{~Gy} / \mathrm{min}$. Resistant cells were generated by mimicking the clinical radiotherapy treatment as previously described (10). Cells that survived irradiation with $60 \mathrm{~Gy}$ (exposure to $2 \mathrm{~Gy}$ 30 times) after 1 month of culture and passaging were used for further analysis and termed '5637R' cells.

Cell cycle analysis. Logarithmic-phase 5637 or $5637 \mathrm{R}$ cells were seeded in $10-\mathrm{cm}$ dishes at $1 \times 10^{7}$ cells/dish and were cultured overnight at $37^{\circ} \mathrm{C}$. Subsequently, cells were subjected to $\gamma$-ray of $2 \mathrm{~Gy}$, which is the dose of radiation commonly used in clinical practice; un-irradiated cells were used as control. After 8 and $24 \mathrm{~h}$ post-ionizing radiation (IR), cells were collected and fixed with $70 \%$ ethanol (pre-chilled to $-20^{\circ} \mathrm{C}$ ) for $2 \mathrm{~h}$ at $4^{\circ} \mathrm{C}$; analysis of DNA content was performed by flow cytometry as previously described (11). A total of 20,000 cells were counted and three independent experiments were performed. Modfit LT 3.1 (Verity Software House, Inc., Topsham, ME, USA) was applied to gate and calculate the proportion of cells in various phases of the cell cycle.

Cell migration assay. Transwell migration of unirradiated 5637 and $5637 \mathrm{R}$ cells was determined using $8.0-\mu \mathrm{m}$ pore size hanging inserts from BD Biosciences (Franklin Lakes, NJ, USA) in 24 well plates. For 5637 and $5637 \mathrm{R}, 1 \times 10^{5}$ cells were seeded in the upper chambers covered with $500 \mu \mathrm{l}$ FBS-free DMEM; 1,000 $\mu 1$ DMEM containing 10\% FBS was added to the lower chambers. Following incubation for $24 \mathrm{~h}$ at $37^{\circ} \mathrm{C}$, the chambers were removed carefully; the chambers were washed three times with PBS (pH 7.4; Beyotime Institute of Biotechnology, Haimen, China). Cells were fixed in $100 \%$ methyl alcohol (Sangon Biotech Co., Ltd., Shanghai, China) at room temperature for $15 \mathrm{~min}$ and the chambers were stained with $0.2 \%$ crystal violet (Sangon Biotech Co., Ltd.) for 30-60 $\mathrm{min}$ at room temperature. When the chambers were dry enough, cells were analyzed under a light microscope (magnification, x100).

Clonogenic survival. Cells sensitive to IR were determined with clone formation assay. Logarithmic-phase 5637 or $5637 \mathrm{R}$ cells were seeded in $3-\mathrm{cm}$ dishes at $5 \times 10^{5}$ cells/dish and were cultured overnight at $37^{\circ} \mathrm{C}$. The logarithmic-phase cells in $3-\mathrm{cm}$ dishes were treated with increasing doses of $\gamma$-ray $(0,2,4,6$ and $8 \mathrm{~Gy})$ at room temperature, at a dose rate of $0.79 \mathrm{~Gy} / \mathrm{min}$. The $0 \mathrm{~Gy}$ irradiated group served as a control group. Following incubating for 0 or $24 \mathrm{~h}$ in the incubator, irradiated cells were trypsinized to a single-cell suspension. Subsequently, according to dose, variable 5637 or $5637 \mathrm{R}$ cell densities ( 0 Gy, 200 cells; 2 Gy, 200 cells; 4 Gy, 400 cells; 6 Gy, 800 cells; and 8 Gy, 1,600 cells) were counted and seeded into 6-cm dishes. After 2 weeks of incubation, the colonies were washed with PBS three times, fixed with $100 \%$ methyl alcohol at room temperature for $15 \mathrm{~min}$. Subsequently, cells were stained with $0.2 \%$ crystal violet for $1 \mathrm{~h}$ at room temperature. When the colonies were sufficiently dried, colonies were counted and the surviving fraction was calculated as described in our previous report (11).

Western blot analysis. Cells in the logarithmic growth phase (5637 and 5637R; $5 \times 10^{5}$ cells/dish) were subjected to increasing doses of $\gamma$-ray $(0,2,4,6$ and $8 \mathrm{~Gy})$. Cellular protein was extracted at 2 and 24 h post-irradiation using a M-PER ${ }^{\mathrm{TM}}$ Mammalian Protein Extraction Reagent (cat. no. 78501; Thermo Fisher Scientific, Inc.) containing 1\% protease inhibitor. Proteins were quantified with a bicinchoninic acid protein assay kit (cat. no. 23227; Thermo Fisher Scientific, Inc.). An equal amount of total protein $(60 \mu \mathrm{g})$ was loaded and fractionated via SDS-PAGE on $12 \%$ gels. Proteins were then transferred to polyvinylidene difluoride (PVDF) membranes $(0.45 \mu \mathrm{m}$; Merck KGaA), which were washed with Tris-buffered saline containing 0.05\% Tween-20 (TBST; Sangon Biotech Co., Ltd.) for $5 \mathrm{~min}$, followed by blocking with $5 \%$ bovine serum albumin (AR2440, Sangon Biotech Co., Ltd.) for $1 \mathrm{~h}$ at room temperature. Subsequently, the membranes were washed with TBST for $10 \mathrm{~min}$ and incubated with primary antibodies at $4^{\circ} \mathrm{C}$ overnight. The PVDF membranes were then washed three times with TBST and incubated with secondary antibodies for $1 \mathrm{~h}$ at room temperature. Finally, the bands were visualized with the ChemiDoc XRS system (Bio-Rad Laboratories, Inc., Hercules, CA, USA). In the present study, the source and use of all mitogen-activated protein kinase (MAPK)-associated antibodies, including anti-extracellular signal-regulated kinase (ERK; 1:1,000; cat. no. ER131218; HuaAn Biotechnology, Inc., Hangzhou, China), c-Jun N-terminal kinase (JNK; 1:1,000; cat. no. sc-571; Santa Cruz Biotechnology, Inc., Dallas, TX, USA), P38 (1:1,000; cat. no. 9212), phosphorylated (p)-ERK $(1: 1,000$, cat. no. 4370), p-JNK (1:1,000; cat. no. 9251), p-P38 (1:2,000; cat. no. 9216; all Cell Signaling Technology, Inc., Danvers, MA, USA) and GADPH (1:1,000; cat. no. AB-P-R; Hangzhou Goodhere Biotechnology Co., Ltd., Hangzhou, China) and horseradish peroxidase-conjugated secondary antibodies against mouse (1:1,000; cat. no. A0216; Beyotime Institute of Biotechnology) or rabbit (1:1,000; cat. no. A0208; Beyotime Institute of Biotechnology), were reported previously (12). Other primary antibodies used were as follows: Anti-ovarian cancer-2/disabled homolog 2 interactive protein (DAB2IP; rabbit polyclonal antibody; 1:650; received from Professor Hsieh, University of Texas Southwestern Medical Center, Dallas, TX, USA), anti-early growth response-1 (EGR-1; 1:1,000; monoclonal antibody; cat. no. ab133695; Abcam, Cambridge, MA, USA), anti- $\beta$-catenin (1:1,000; monoclonal antibody; cat. no. 610153; BD Biosciences, San Jose, CA, USA), anti-signal transducer and activator of transcription 3 (STAT3; monoclonal antibody; 1:1,000; cat. no. ET-1607-38; HuaAn Biotechnology, Inc.), anti-phosphorylated (p)-STAT3 (Tyr-727; 1:1,000; monoclonal antibody; cat. no. ET1607-39, HuaAn Biotechnology, Inc.), anti-phospho-STAT3 (Ser-705) (1:1,000; monoclonal antibody; cat. no. ET1603-40; Hangzhou HuaAn Biotechnology Co., Ltd., Hangzhou, China), anti-vimentin (1:500; monoclonal antibody; cat. no. EM0401; Hangzhou HuaAn Biotechnology Co., Ltd.), and anti-E-cadherin (1:500; monoclonal antibody; cat. no. EM0502; Hangzhou HuaAn Biotechnology Co., Ltd.). The protein bands were visualized using the BeyoECL Plus 
A

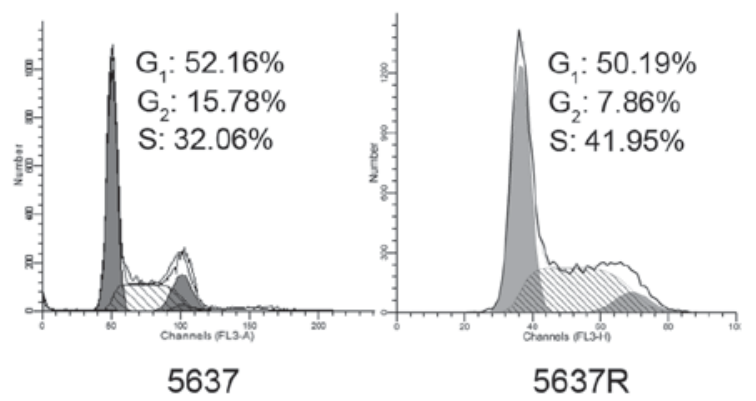

B

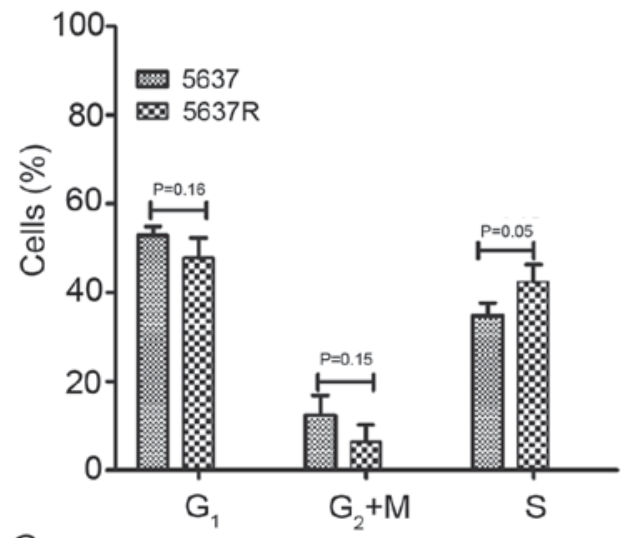

C

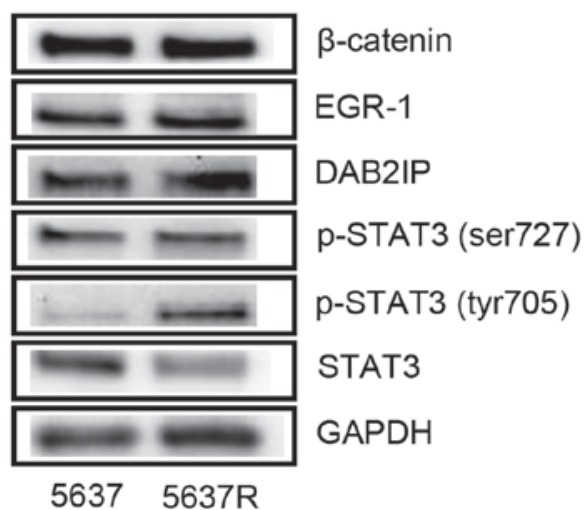

Figure 1. Fractionated irradiation increases $\mathrm{S}$ phase cell distribution and STAT3 phosphorylation in BCa cells. Human BCa cell line, 5637, underwent fractionated irradiation with 2 Gy for 30 times and continuously cultured for 1 month; cells that survived were denoted as 5637R. (A) Cell cycle distribution in 5637 and $5637 \mathrm{R}$ cells was assayed by flow cytometry analysis. (B) Percentage of cells in different phases was compared. The results are the averages of triplicate experiments and error bars represent the standard error. (C) Expression of a series of tumor-associated proteins as detected by western blotting. GADPH was loaded as an internal control $\mathrm{BCa}$, bladder cancer; 5637R, radioresistant 5637 cells; EGR-1, early growth response-1; DAB2IP, ovarian cancer-2/disabled homolog 2 interactive protein; p, phosphorylated; STAT3, signal transducer and activator of transcription 3.

kit (cat. no. P0018; Beyotime Institute of Biotechnology) and the ChemiDoc XRS system (Bio-Rad Laboratories, Inc.).

Statistical analysis. Data were presented as the mean \pm standard error of at least three independent experiments. The results were tested for significance using an unpaired Student's t-test. Statistical analysis was conducted using STATA 10.0 statistics software (StataCorp LP, College Station, TX, USA) and $\mathrm{P}<0.05$ was considered to indicate a statistically significant difference.

\section{Results}

FI increases $S$ phase cell distribution and STAT3 phosphorylation in $B C a$ cells. In the present study, a human $\mathrm{BCa}$ cell line, 5637 was exposed 30 FI treatments of 2 Gy/day. Following 1 month of culturing and passaging, surviving cells were named 5637R. Initially, the present study investigated the effects of FI on the cell cycle distribution on 5637 and 5637R cells. An increased population of $\mathrm{S}$ phase cells was detected in the 5637R group compared with 5637 cells (Fig. 1A and $\mathrm{B}$ ). The proportion of $\mathrm{S}$ phase cells in $5637 \mathrm{R}$ vs. 5637 was $42.9 \pm 4.6$ vs. $33.8 \pm 2.5 \%$, respectively $(\mathrm{P}=0.05$; mean \pm standard error, data from three independent experiments). Subsequently, the expression of a series of proteins associated with FI treatment (10) was analyzed, including $\beta$-catenin, EGR-1, DAB2IP and STAT3 and detected via western blot analysis. It was revealed that STAT3 phosphorylation (Tyr705) was notably increased in 5637R cells compared with 5637 cells (Fig. 1C). Tyr705 phosphorylation is a key event required for STAT-3 activation, which induces the upregulation of various genes involved in cell survival and proliferation (13). Therefore, it was hypothesized that, compared with 5637 cells, the increased expression of p-STAT3 (Tyr705) in 5637R cells may confer a survival advantage in response to IR, due to the promotion of cell survival and evasion of apoptosis. Conversely, visible alterations in the expression of p-STAT3 (Ser727) were not observed in 5637R cells. Furthermore, it was revealed that the expression of STAT3 was lower in 5637R cells than in 5637 cells. Notably, the STAT3 antibody used in the present study does not recognize p-STAT3; therefore, it cannot be concluded that STAT3 synthesis is decreased in 5637R cells compared with in 5637 cells from this result. In addition, no differences were observed in $\beta$-catenin, EGR- 1 and DAB2IP expression between the 5637 and 5637R cells.

FI enhances BCa cells migration ability. In the migration assay, 5637R cells demonstrated notable increases in migratory ability compared with in 5637 cells (Fig. 2A). The number of migratory cells was significantly increased in the 5637R cell group compared with in 5637 cells $(689.6 \pm 24.05$ vs. $346.4 \pm 72.64$, respectively, $\mathrm{P}=0.002$; Fig. $2 \mathrm{~B}$ ). The present study also investigated the expression of epithelial-mesenchymal transition (EMT)-associated markers, including E-cadherin and vimentin in 5637 and 5637R cells. The results revealed that 5637 and 5637R cells exhibited a high expression of E-cadherin; however, the expression of vimentin was too low for detection in the present study. In addition, no notable variations in the expression of the aforementioned protein were observed between 5637 and $5637 \mathrm{R}$ cells.

FI enhances BCa cells radioresistance associated with activation of the ERK/MAPK signaling pathway post-IR. To explore the effects of FI on BCa cells, 5637 and 5637R cells were subjected to increasing doses of IR (0, 2, 4, 6 and $8 \mathrm{~Gy})$. Clonogenic survival analysis demonstrated that 5637R cells, which survived FI exposure, exhibited increased resistance to IR compared with the parental cell line, 5637 (Fig. 3A). The 

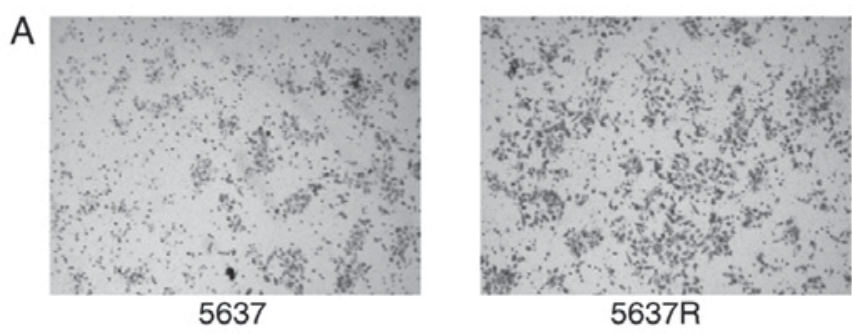

C

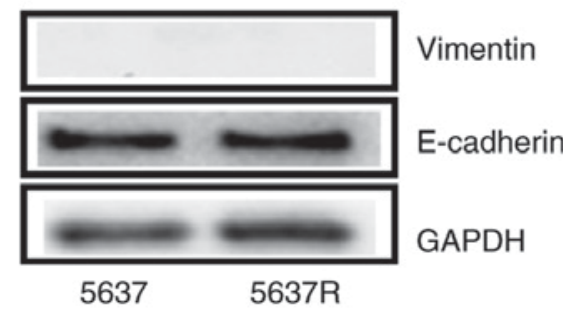

B

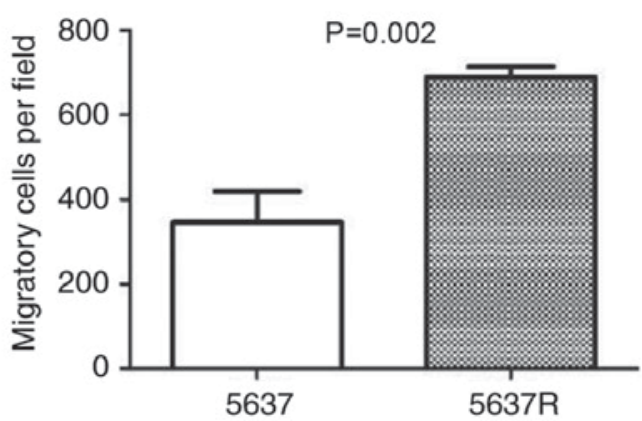

Figure 2. Fractionated irradiation enhances the migration ability of bladder cancer cells. (A) Representative images and (B) quantification of Transwell migration analysis of 5637 and 5637R cells. The data are presented as the mean \pm standard error of triplicate experiments. (C) Expression of migration-associated proteins was detected by western blotting. GADPH was loaded as an internal control. 5637R, radioresistant 5637 cells.
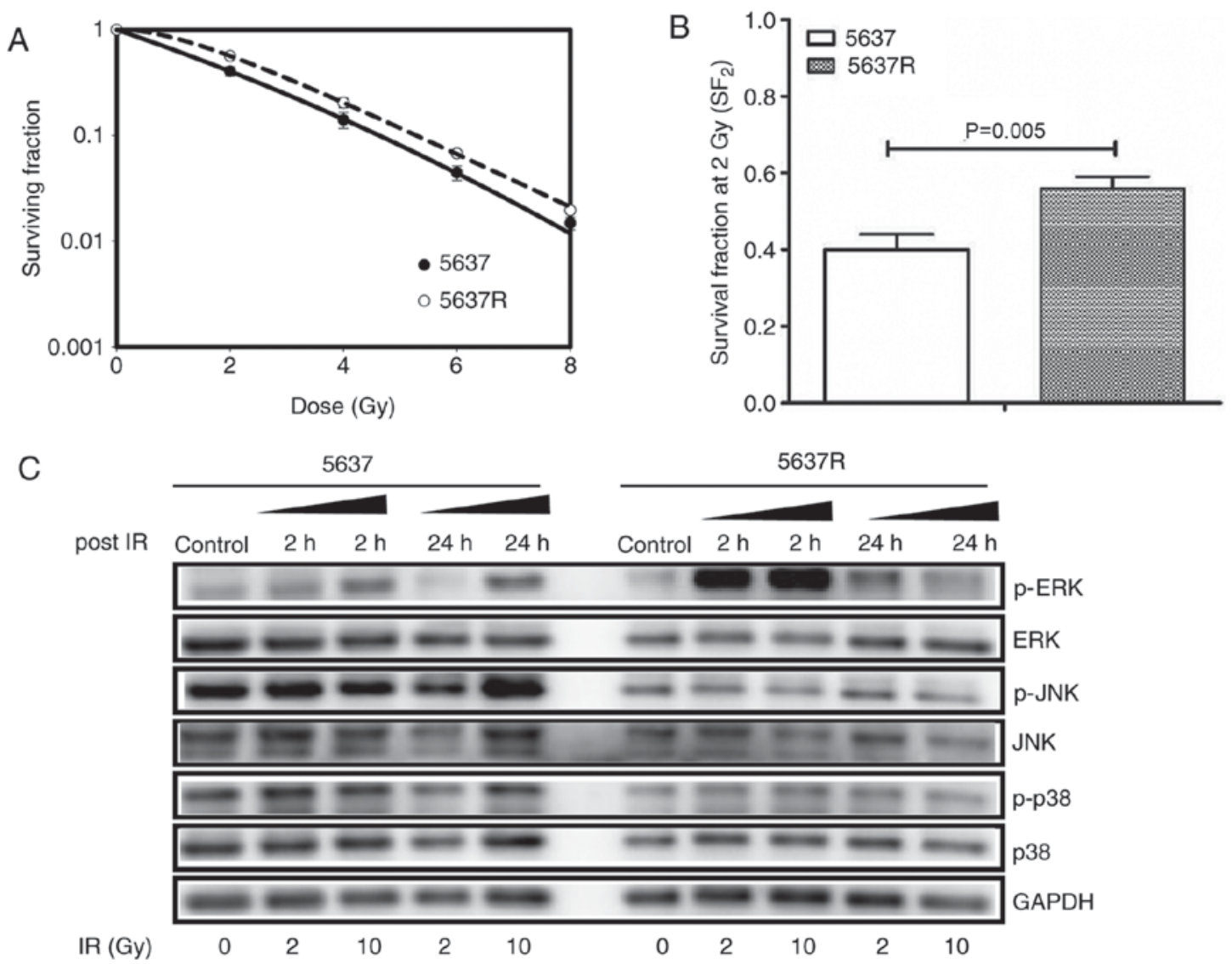

Figure 3. Fractionated irradiation enhances the radioresistance of bladder cancer cells associated with activation of the ERK/mitogen-activated protein kinase pathway post-IR. (A) Radiosensitivity of 5637 and 5637R cells was detected via a colony formation assay. (B) The surviving fraction of 5637R and 5637 cells treated with $2 \mathrm{~Gy}$ were compared. The data are presented as the mean \pm standard error of triplicate experiments. (C) Cells were treated with increasing doses of IR (0, 2 and $10 \mathrm{~Gy}$, respectively) and the samples were collected at the time points as indicated. The expression of ERK, p-ERK, P38, p-P38 and JNK were detected via western blot analysis. GADPH was loaded as an internal control. 5637R, radioresistant 5637 cells; p, phosphorylated; ERK, extracellular signal-regulated kinase; JNK, c-Jun N-terminal kinases; IR, ionizing radiation.

surviving fraction of 5637R and 5637 cells treated with $2 \mathrm{~Gy}$ were $0.56 \pm 0.03$ and $0.40 \pm 0.04$, respectively $(\mathrm{P}=0.005$; Fig. $3 \mathrm{~B})$.
In order to further understand the molecular mechanism underlying FI-induced radioresistance, activation of the 

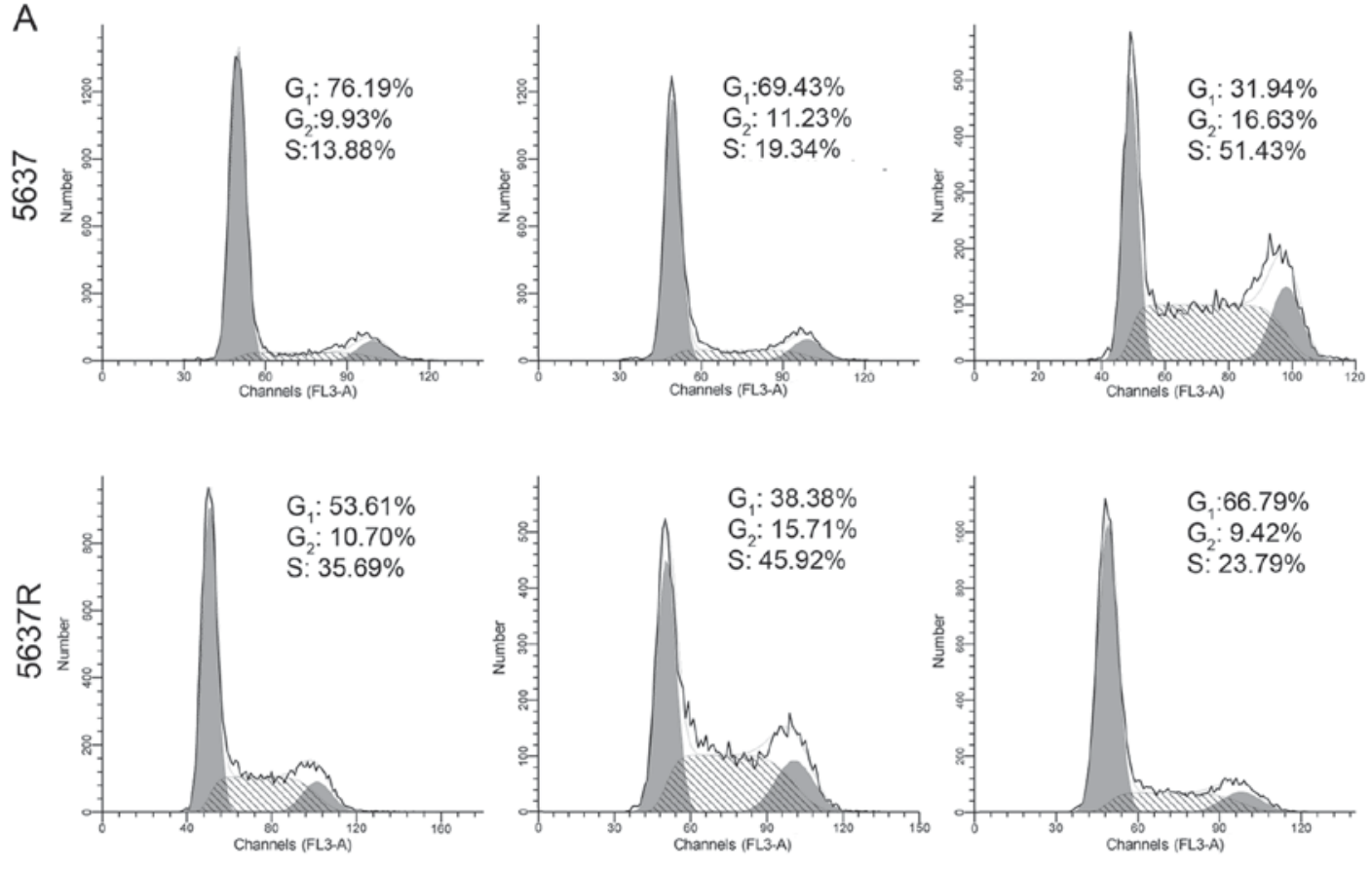

Control

$8 \mathrm{~h}$ post-IR

$24 \mathrm{~h}$ post-IR

B

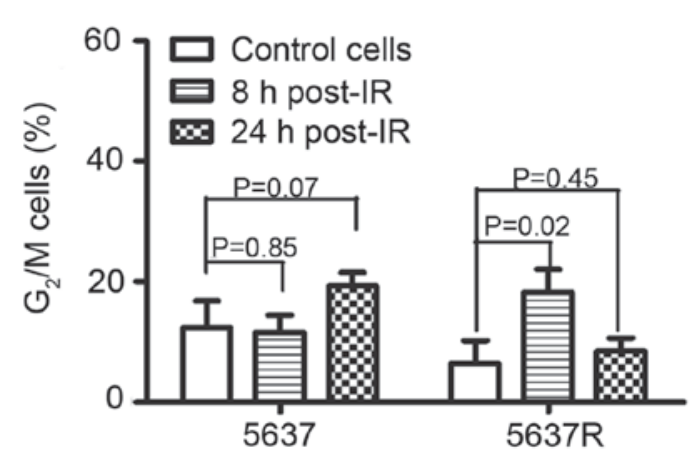

Figure 4. IR induces $\mathrm{G}_{2} / \mathrm{M}$ inhibition in bladder cancer cells. 5637 and $5637 \mathrm{R}$ cells were treated with $2 \mathrm{~Gy}$ and collected at 0,8 and $24 \mathrm{~h}$ following radiation. Propidium iodide staining was applied to detect the distribution of cell cycle response to IR by flow cytometry. DNA profiles were obtained at the time points as indicated. (A) Representative images of cell cycle distribution and (B) average percentage of cells in $\mathrm{G}_{2} / \mathrm{M}$ phase. The percentage of cells in $\mathrm{G}_{2} / \mathrm{M}$ phase was increased by $\sim 2$-fold in $5637 \mathrm{R}$ cells $(6.42 \pm 3.82$ to $18.23 \pm 3.85 \%) 8 \mathrm{~h}$ post-IR. For 5637 cells, $\mathrm{G}_{2} / \mathrm{M}$ arrest occurred within $24 \mathrm{~h}$ post-IR; the percentage of cells in $\mathrm{G}_{2} / \mathrm{M}$ phase was increased from $12.36 \pm 4.45 \%$ in the control group to $19.26 \pm 2.21 \%$ in IR-treated cells. The results are the average of triplicate experiments and error bars represent standard error.

MAPK signaling pathway and the cell cycle distribution in response to IR were detected via western blot analysis and flow cytometry, respectively. The present study reported that 5637R cells exhibited an increase in ERK phosphorylation $2 \mathrm{~h}$ post IR, which was not observed in 5637 cells (Fig. 3C). As presented in Fig. 4, irradiation induced a notable $\mathrm{G}_{2} / \mathrm{M}$ arrest in 5637R cells $8 \mathrm{~h}$ post-IR; the percentage of cells in $\mathrm{G}_{2} / \mathrm{M}$ phase was increased from $6.42 \pm 3.82$ to $18.23 \pm 3.85 \%$ compared with in the control group. Conversely, $\mathrm{G}_{2} / \mathrm{M}$ arrest was observed in 5637 cells 24 h following radiation exposure; the percentage of cells in $\mathrm{G}_{2} / \mathrm{M}$ phase was increased from $12.36 \pm 4.45$ to $19.26 \pm 2.21 \%$ compared with in the control group. Collectively, these results indicated that the $\mathrm{G}_{2} / \mathrm{M}$ checkpoint and the robust ERK/MAPK signaling pathway may serve roles in the increased radioresistance exhibited by $5637 \mathrm{R}$ cells compared with in 5637 cells.

\section{Discussion}

External beam radiation therapy with daily fractionation of 1.8-2 Gy over a period of several weeks is a standard treatment option in clinical radiotherapy; however, due to idiopathic or acquired radioresistance (ARR), not all patients benefit from radiotherapy (14). Differing from that of congenital radiation tolerance, adaptive resistance to radiotherapy occurs during the course of FI in numerous patients, resulting in tumor metastasis and recurrence, which comprises ARR (15). This may be the main reason for the failure of cancer treatment with radiotherapy; however, the mechanism underlying the development of ARR remains poorly understood.

In order to identify biomolecules involved in ARR, the present study reproduced the phenomenon of radiation tolerance induced by FI to obtained resistant 5637R cells. These cells may be a useful cell model to investigate the 
molecular mechanism underlying ARR. Compared with in 5637 cells, an increase in the proportion of 5637R cells in $\mathrm{S}$ phase was observed, along with enhanced migration ability and elevated levels of STAT3 phosphorylation. It has been reported that cells in late $\mathrm{S}$ phase are relatively insensitive to IR compared with cells of other phases (16). In addition, an increased population of $\mathrm{S}$ phase 5637R cells may indicate high levels of cellular division and proliferation. Thus, the present study suggested that an increased proportion of FI-treated cells in S-phase cells may be associated with IR tolerance exhibited by $5637 \mathrm{R}$ cells compared with 5637 cells.

Additionally, in the present study, 5637R cells exhibited increased migration abilities compared with 5637 cells as determined by a Transwell assay. To determine whether the increased migration abilities observed in FI-resistant cells may be associated with EMT, the expression of EMT markers, including E-cadherin, vimentin and $\beta$-catenin was detected within $\mathrm{BCa}$ cells; however, the expression of these proteins was similar in 5637 and $5637 \mathrm{R}$ cells, suggesting that $5637 \mathrm{R}$ may exhibit the characteristics of epithelial cells. Conversely, compared with in 5637 cells, a notable elevation of p-STAT3 (Tyr705) was detected in 5637R cells in the present study. It has been reported that STAT3 phosphorylation at Tyr705 is critical for the growth and survival of BCa cells (17). This may promote the migration and invasion of BCa cells (18). Therefore, the present study proposed that increased mobility and S-phase cell distribution demonstrated by 5637R cells may be partly due to STAT3 activation; however, the upstream molecular mechanism that activates STAT3 following FI requires further investigation.

MAPK signaling pathways regulate the expression of genes associated with proliferation, differentiation and apoptosis, and responses to environmental changes in eukaryotic cells (19). There are 14 MAPK-associated genes in mammals, which are characterized into 7 groups; ERK1/2, JNK1/2/3 and P38 isoforms are the three most notable and widely researched protein kinases (20). In the present study, variations in the response of the MAPK pathway to IR were compared between parental 5637 cells and their FI-treated derivatives (5637R). The results of the present study revealed that irradiation markedly upregulated ERK phosphorylation in 5637R cells compared with in 5637 cells. In addition, in 5637R cells treated with 2 Gy, ERK activation via phosphorylation in response to IR continued to increase within $24 \mathrm{~h}$ post-irradiation. The ERK cascade has been associated with cell survival and proliferation (21). Furthermore, ERK can activate a series of transcription factors, such as STAT3, resulting in gene regulation, which affects cell cycle progression, cell motility and apoptosis (22). Therefore, the present study investigated whether ERK phosphorylation may be the key event inducing the activation of STAT3. Compared with in 5637 cells, 5637R cells exhibited notably low basal expression of p-ERK. A correlation between STAT3 activation and ERK1/2 inhibition has also been reported in $\mathrm{BCa}$ cells in response to long-term nicotine exposure (23).

To understand the mechanism underlying increased STAT3 phosphorylation associated with $\mathrm{BCa}$, studies have investigated the phosphoinositide 3-kinase/protein kinase $\mathrm{B}$ (Akt)/STAT3 and the Janus kinase (JAK)/STAT3 signaling pathways to determine the proteins upstream of STAT3 in radioresistant $5637 \mathrm{R}$ cells $(18,24)$. The results of the present study revealed that $5637 \mathrm{R}$ cells possess higher levels of p-JAK2 compared with in 5637. No significant difference in the expression levels of p-Akt between 5637 and 5637R cells (data not shown). Therefore, the present study suggested that abnormal activation of the JAK2/STAT3 signaling pathway may be associated with malignant phenotypes of 5637R cells, including increased migration ability and a higher proportion of S-phase cells. However, further investigation is required to understand whether the mechanism underlying persistent activation of the JAK2/STAT3 signaling pathway is associated with FI. Additionally, the association between JAK2/STAT3 and ERK requires further investigation.

Collectively, the findings of the present study suggested that increased activation of STAT3 and robust ERK/MAPK signaling may confer survival advantages in 5637R cells and increased resistance to radiotherapy. Furthermore, the present study proposed that $\mathrm{p}$-STAT3 may be considered as a potential biomarker to detect radioresistance and tumor recurrence of patients with $\mathrm{BCa}$ following conventional radiotherapeutic intervention. Additionally, co-treatment with an ERK inhibitor may be a viable approach to improve the anticancer efficacy of radiotherapy in patients with $A R R$.

\section{Acknowledgements}

Not applicable.

\section{Funding}

The present study was supported by the National Nature Science Foundation of China (grant nos. 31870846 and 31270896), the Natural Science Foundation of Shanghai (grant nos. 18ZR1403600 and 11ZR1402100), the Scientific Research Foundation for the Returned Overseas Chinese Scholars, State Education Ministry (grant no. 44-8) and the Zhuoxue Project of Fudan University.

\section{Availability of data and materials}

The datasets used and/or analyzed during the current study are available from the corresponding author on reasonable request.

\section{Authors' contributions}

ZK designed the experiments. GM performed the experiments. ZK, GM and YY analyzed the data. ZK and GM wrote and revised the manuscript. All authors reviewed the manuscript.

\section{Ethics approval and consent to participate}

Not applicable.

\section{Patient consent for publication}

Not applicable.

\section{Competing interests}

The authors declare that they have no competing interests. 


\section{References}

1. Siegel RL, Miller KD and Jemal A: Cancer statistics, 2017. CA Cancer J Clin 67: 7-30, 2017.

2. Miyake M, Morizawa Y, Hori S, Tatsumi Y, Onishi S, Owari $\mathrm{T}$, Iida $\mathrm{K}$, Onishi $\mathrm{K}$, Gotoh $\mathrm{D}$, Nakai Y, et al: Diagnostic and prognostic role of urinary collagens in primary human bladder cancer. Cancer Sci 108: 2221-2228, 2017.

3. Chang Y, Xu J and Zhang Q: Microplate magnetic chemiluminescence immunoassay for detecting urinary survivin in bladder cancer. Oncol Lett 14: 4043-4052, 2017.

4. Li X, Wang Y, Xu J and Zhang Q: Sandwich ELISA for detecting urinary Survivin in bladder cancer. Chin J Cancer Res 25: 375-381, 2013.

5. Ogihara K, Kikuchi E, Yuge K, Ito Y, Tanaka N, Matsumoto K, Miyajima A, Asakura $\mathrm{H}$ and Oya M: Refraining from smoking for 15 years or more reduced the risk of tumor recurrence in non-muscle invasive bladder cancer patients. Ann Surg Oncol 23: 1752-1759, 2016.

6. Nam JK, Park SW, Lee SD and Chung MK: Prognostic value of sex-hormone receptor expression in non-muscle-invasive bladder cancer. Yonsei Med J 55: 1214-1221, 2014.

7. Chou R, Selph SS, Buckley DI, Gustafson KS, Griffin JC, Grusing SE and Gore JL: Treatment of muscle-invasive bladder cancer: A systematic review. Cancer-Am Cancer Soc 122: 842-851, 2016.

8. Soloway MS: ICUD-EAU International consultation on bladder cancer 2012: Recommendations on bladder cancer-progress in a cancer that lacks the limelight. Eur Urol 63: 1-3, 2013.

9. Bloom HJ, Hendry WF, Wallace DM and Skeet RG: Treatment of T3 bladder cancer: Controlled trial of pre-operative radiotherapy and radical cystectomy versus radical radiotherapy. Br J Urol 54: 136-151, 1982.

10. Chang R, He H, Mao G and Kong Z: Upregulating DAB2IP expression via EGR-1 inhibition, a new approach for overcoming fractionated-irradiation-induced cross-tolerance to ionizing radiation and mitomycin $\mathrm{C}$ in tumor cells. Int $\mathbf{J}$ Radiat Biol 93: 386-393, 2017.

11. Kong Z, Xie D, Boike T, Raghavan P, Burma S, Chen DJ, Habib AA, Chakraborty A, Hsieh JT and Saha D: Downregulation of human DAB2IP gene expression in prostate cancer cells results in resistance to ionizing radiation. Cancer Res 70: 2829-2839, 2010.
12. He H, Chang R, Zhang T, Yang $\mathrm{C}$ and Kong Z: ATM mediates DAB2IP-deficient bladder cancer cell resistance to ionizing radiation through the $\mathrm{p} 38 \mathrm{MAPK}$ and $\mathrm{NF}-\kappa \mathrm{B}$ signaling pathway. Mol Med Rep 16: 1216-1222, 2017.

13. Bhattacharya S, Ray RM and Johnson LR: STAT3-mediated transcription of Bcl-2, Mcl-1 and c-IAP2 prevents apoptosis in polyamine-depleted cells. Biochem J 392: 335-344, 2005.

14. Shimura T: Acquired radioresistance of cancer and the AKT/GSK3//cyclin D1 overexpression cycle. J Radiat Res 52: 539-544, 2011.

15. Shimura T: Targeting the AKT/cyclin D1 pathway to overcome intrinsic and acquired radioresistance of tumors for effective radiotherapy. Int J Radiat Biol 93: 381-385, 2017.

16. Pawlik TM and Keyomarsi K: Role of cell cycle in mediating sensitivity to radiotherapy. Int J Radiat Oncol Biol Phys 59: 928-942, 2004.

17. Chen CL, Cen L, Kohout J, Hutzen B, Chan C, Hsieh FC, Loy A, Huang V, Cheng G and Lin J: Signal transducer and activator of transcription 3 activation is associated with bladder cancer cell growth and survival. Mol Cancer 7: 78, 2008.

18. Yang C, Zhang W, Wang L, Kazobinka G, Han X, Li B and Hou T: Musashi-2 promotes migration and invasion in bladder cancer via activation of the JAK2/STAT3 pathway. Lab Invest 96: 950-958, 2016.

19. Raman M, Chen W and Cobb MH: Differential regulation and properties of MAPKs. Oncogene 26: 3100-3112, 2007.

20. Cargnello $M$ and Roux PP: Activation and function of the MAPKs and their substrates, the MAPK-activated protein kinases. Microbiol Mol Biol Rev 75: 50-83, 2011.

21. Roskoski R Jr: ERK1/2 MAP kinases: Structure, function, and regulation. Pharmacol Res 66: 105-143, 2012.

22. Sebolt-Leopold JS and Herrera R: Targeting the mitogen-activated protein kinase cascade to treat cancer. Nat Rev Cancer 4: 937-947, 2004.

23. Chen RJ, Ho YS, Guo HR and Wang YJ: Long-term nicotine exposure-induced chemoresistance is mediated by activation of Stat 3 and downregulation of ERK1/2 via nAChR and beta-adrenoceptors in human bladder cancer cells. Toxicol Sci 115: 118-130, 2010.

24. Li Y, Guo G, Song J, Cai Z, Yang J, Chen Z, Wang Y, Huang Y and Gao Q: B7-H3 promotes the migration and invasion of human bladder cancer cells via the PI3K/Akt/STAT3 signaling pathway. J Cancer 8: 816-824, 2017. 